\title{
Aggregated States of Molecular Chains in Solid-State Films of Poly $(\gamma$ - $n$-alkyl D-glutamate) Cast from Various Solutions
}

\author{
Ken'ichi Ito, Tisato KajIyama, and Motowo Takayanagi \\ Department of Applied Chemistry, Faculty of Engineering, \\ Kyushu University, Fukuoka 812, Japan.
}

(Received December 1, 1976)

\begin{abstract}
The aggregated states of molecular chains in the crystalline region were studied for well annealed films of poly( $\gamma$-methyl D-glutamate) and poly $(\gamma-n$-hexyl D-glutamate) cast from various solutions. These crystalline regions were found to be composed of the $\alpha$-helical chains in hexagonal array by means of the X-ray diffraction technique. Both a planar and a (1010) uniplanar orientations were found in the solid films of poly( $\gamma$-methyl D-glutamate), depending upon the kind of solvents and casting temperatures. Only a (1120) uniplanar orientation was observed in the solid film of poly( $\gamma$-n-hexyl D-glutamate). Viscosity measurements and optical microscopic observations revealed that the aggregated states of molecular chains in the solid were in close relation to those in solutions found in the evaporating process of solvents. In the case of poly( $\gamma$-methyl D-glutamate), a planar orientation was observed when a cholesteric solution was cast and dried, and also a (1010) uniplanar orientation was observed when a solution was gelatinized. On the other hand, in the case of poly $(\gamma-n$-hexyl D-glutamate), a liquid crystalline state was observed in any one solvent used for this report, resulting in a (1120) uniplanar orientation in the cast film.

KEY WORDS Poly $(\gamma$-methyl D-glutamate $) /$ Poly $(\gamma$-n-hexyl D-glutamate) / Planar Orientation / Uniplanar Orientation / Viscosity / Cholesteric Liquid Crystal / Polarizing Micro-Photograph /
\end{abstract}

There have been many investigations on the aggregated states of poly( $\gamma$-benzyl L-glutamate) (PBLG) molecular chains in dilute solutions, concentrated solutions and a solid-state. Doty, et al., ${ }^{1}$ reported that the molecular association of PBLG was of the end-to-end type in a dilute solution of chloroform, dioxane, or benzene. They also mentioned that in the dimethylformamide solution, the association of the $\alpha$-helices was prevented by solvation of helix-ends with dimethylformamide molecules. Wada, et al., ${ }^{2}$ reported that PBLG molecules existed in a dispersed state in a dilute dimethylformamide solution, whereas in a dilute solution of dimethylformamide-dioxane, associations of headto-tail and side-by-side types were formed with decreasing the concentration of PBLG. Robinson, et al., ${ }^{3}$ reported that $\mathrm{PBLG}$ and poly $(\gamma$-methyl L-glutamate) (PMLG) molecules formed a cholesteric liquid crystal in concentrated solutions of dioxane, $m$-cresol, chloroform and so on. In these solutions, the two characteristic concentrations were found, that is, the A-point above which the anisotropic phase appears and the Bpoint above which the isotropic liquid phase no longer exists. These A- and B-points depend on molecular weight. The phase separation at the A-point is predicted by Flory's theory. ${ }^{4}$ Hermans $^{5}$ studied the viscosity-concentration relationship for a concentrated poly $(\gamma$-benzyl Lglutamate) solution in $m$-cresol using a shear type viscometer and reported that below the Apoint, the viscosity at low shear stress increased with increasing concentration and above the A-point, it decreased with increasing concentration. Tobolsky, et al., ${ }^{6}$ reported that both the mesomorphic and the paracrystalline regions were formed in the solid-state films of PBLG cast from a solution in chloroform. In the former case, the $\alpha$-helices lay parallel with each 
other in the plane with the small helical twist relative to one another around the axis perpendicular to the film surface. In the latter case, the molecular chains are aligned parallel in a certain plane and packed in a slightly distorted hexagonal arrangement, in such a way that the neighboring planes do not show the helical twist.

In the investigations mentioned above, they discussed the aggregated states of the molecular chains or the molecular chain orientation in the only one state such as a dilute solution, a concentrated solution, or a solid film. It comes up as an important problem to study the variation of way of molecular aggregation in the successive change of state from a dilute solution to a solid film, which may depend on the kind of solvents, temperature, molecular weight, and so forth. Then, it is interesting to investigate how the molecular aggregation of the $\alpha$-helical molecular chains in a concentrated solution such as a liquid crystalline structure is reflected in the solid-state. In this paper, we will discuss the relationship between the aggregated states of molecular chains of poly $(\gamma$ - $n$-alkyl D-glutamate) (PnADG) having different side chain lengths in the solutions and those in the solids cast from various solutions of $\alpha$-helix forming solvents. The X-ray diffraction studies, the optical microscopic observations and the viscosity measurements were used for this purpose.

\section{EXPERIMENTAL}

Poly $(\gamma-n$-alkyl D-glutamate (PnADG) with side chains of various lengths were prepared by the alcoholysis method from poly $(\gamma$-methyl D-glutamate) (AJICOAT A-200, $\bar{M}_{v}=140,000$ ) as a starting material, using ethanol, propanol, butanol, and hexanol. ${ }^{7}$ The samples of poly $(\gamma$-ethyl Dglutamate) (PEDG), poly( $\gamma$-n-propyl D-glutamate) (PnPDG), poly $(\gamma-n$-butyl D-glutamate) (PnBDG) and poly $(\gamma-n$-hexyl D-glutamate) (PnHDG) were

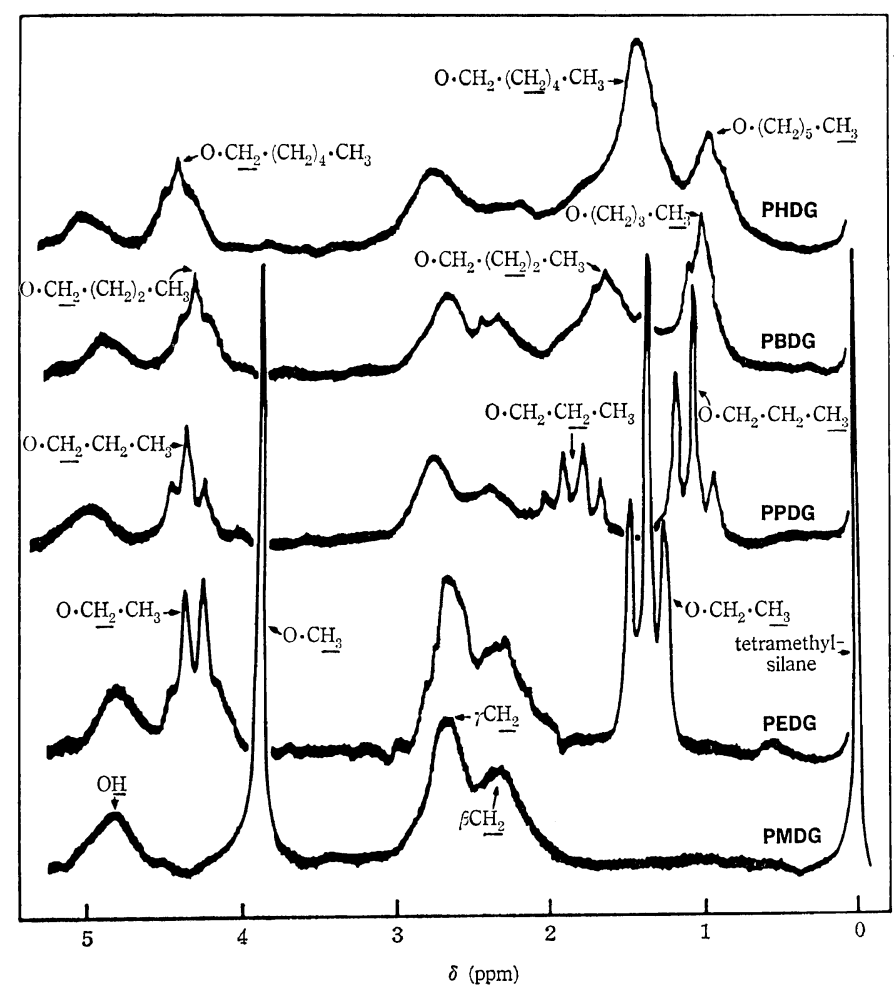

Figure 1. High resolution NMR spectra of PnADG in a trifluoroacetic acid solution. Assignments of various peaks are designated by underlined chemical formulas. 
obtained by this method. The degree of substitution of the methyl group for the $n$-alkyl group was evaluated by the high resolution NMR spectra (model JNM-MH-60E, Japan Electron Optics Laboratory Co., Ltd.) as shown in Figure 1. In this paper we chose PMDG and PnHDG with the side chains of the shortest and the longest lengths respectively, as the representatives of PnADG.

The PMDG films with $0.2-0.8 \mathrm{~mm}$ in thickness were prepared from various solvents such as chloroform, 1,2-dichloroethane, 1,1,2-trichloroethane, 1,2,3-trichloropropane, cis-dichloroethylene, trans-dichloroethylene, trichloroethylene, chlorobenzene, $N, N$-dimethylformamide, pyridine, or dioxane. The PnHDG films were prepared from chloroform, 1,2-dichloroethane, trichloroethylene, or benzene. In the case of trichloroethylene, chlorobenzene, $N, N$-dimethylformamide, pyridine, and dioxane, PMDG solutions were prepared by heating above $333 \mathrm{~K}$ and cooling down to room temperature. The other solutions were prepared at room temperature. The solid-state films of PMDG and PnHDG were cast by evaporating solvents within a period from a week to three months at room temperature. These solid-state films were dried in vacuo at room temperature for about a week and then, the PMDG films were annealed at $488 \mathrm{~K}$ for $1 \mathrm{hr}$ in vacuo.

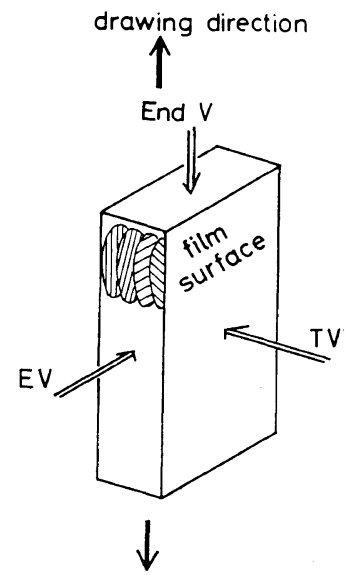

Figure 2. Directions of the incident X-ray beam; normal, parallel to the film surface and along the drawing direction are designated by through (TV), edge on (EV) and end (End V) views, respectively.
The wide angle $\mathrm{X}$-ray photographs of these specimens were taken at room temperature with a rotating anode unit (Rotaunit RU-3, Rigaku Denki Co.). The X-ray beams $(\mathrm{Cu}-\mathrm{K} \alpha$, Ni filter $)$ were normal to the film surface (TV) or along the edge direction (EV). For the drawn samples, the X-ray beam was also irradiated along the drawing direction (End V) as shown in Figure 2 other than the directions of TV and EV.

Solution viscosities were measured at $313 \mathrm{~K}$ using a Pipette-capillary and a uniform bore type viscometers constructed in our laboratory. ${ }^{8}$

The concentrated solutions of PMDG and PnHDG in various solvents were poured into the glass cell having a Teflon spacer with a thickness of $1.1 \mathrm{~mm}$, and their polarizing microphotographs were taken under the cross nicols at room temperature.

\section{RESULTS AND DISCUSSION}

Aggregated State and Orientation of Molecular Chains in Poly( $\gamma$-methyl D-glutamate)

The X-ray photographs for the solid-state films of poly $(\gamma$-methyl D-glutamate) (PMDG) were roughly classified into two groups on the basis of X-ray diffraction pattern types, which depend on the kind of solvents used. Solvents of chloroform, 1,2-dichloroethane, 1,1,2-trichloroethane, 1,2,3-trichloropropane, cis-dichloroethylene, and trans-dichloroethylene, belong to Group 1 , and those of trichloroethylene, chlorobenzene, pyridine, $N, N$-dimethylformamide, and dioxane belong to Group 2. In Table I, the classification of solvents are listed.

Figure 3 shows the X-ray through view (TV) and edge view (EV) photographs for the annealed PMDG solid film cast from a solution in chloroform (Group 1). The PMDG films cast from the other solvents which belong to Group 1 exhibit the similar X-ray diffraction patterns as in Figure 3. Figure 4 shows the $\mathrm{X}$-ray TV and EV photographs for the PMDG film cast from a solution in trichloroethylene (Group 2), and the similar X-ray photographs are taken for the PMDG films cast from the other solvents in Group 2. First, we will discuss the molecular chain orientations in the solid-state films cast from solutions in Group 1 and Group 2 solvents. 
The X-ray TV diffraction pattern in Figure 3 almost satisfies the hexagonal packing of the $\alpha$ helical chains, with the crystallographic constants of $a=b=1.19 \mathrm{~nm}$ and $c=2.7 \mathrm{~nm}$, as reported by Bamford, et al. ${ }^{9}$ The sharp Debye rings of the TV photograph indicate that there exists a crystalline region composed of $\alpha$-helical chains and also, the $\alpha$-helical axes in the crystalline region are oriented statistically at random with respect to the TV direction. The X-ray EV photograph in Figure 3 shows arcs, whose indices are schematically represented in Figure 5. Figure 3 indicates that the $\alpha$-helical chains exhibit random orientation along the TV direction, whereas a certain kind of orientation exists along the EV direction. The manner of the $\alpha$-helical chain alignment or orientation may be explained by the three characteristic rotational parameters

Table I. Solvents used for poly( $\gamma$-methyl D-glutamate)

\begin{tabular}{llcc}
\hline Solvents & $\begin{array}{c}\text { Dielectric } \\
\text { constants }\end{array}$ & Orientations \\
\hline & Chloroform & 4.8 & \\
1,2-Dichloroethane & 10.4 & \\
Group 1 $1,1,2$-Trichloroethane & - & Planar \\
& 1,2,3-Trichloropropane & - & Orientation \\
& cis-Dichloroethylene & 9.2 & \\
& trans-Dichloroethylene & 2.1 & \\
\hline \multirow{5}{*}{ Group 2 2 Dimethylformamide } & 3.4 & \\
& Trichloroethylene & 5.7 & Uniplanar \\
& Chloridine & 37.6 & Orientation \\
& Dioxane & 12.3 & \\
\hline
\end{tabular}

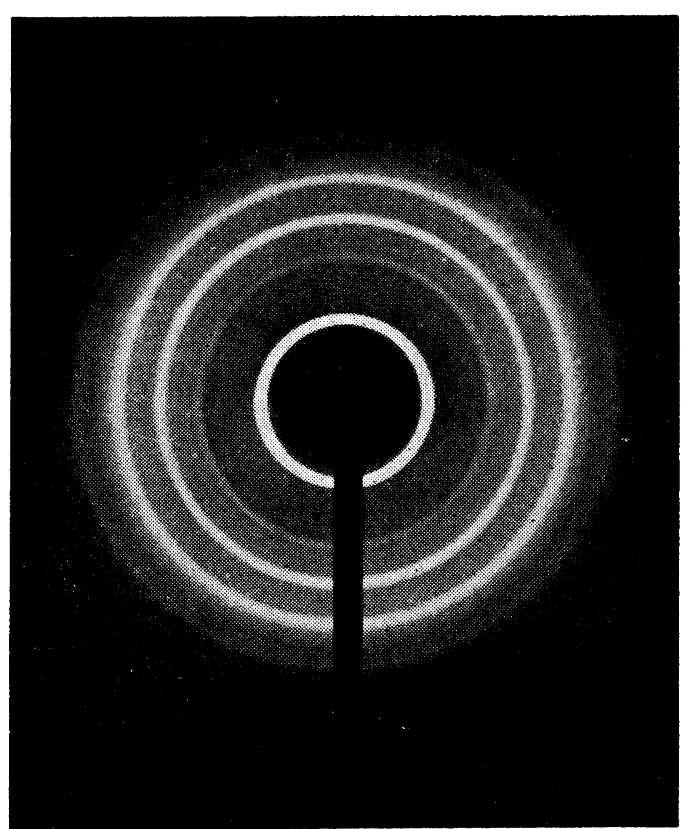

TV

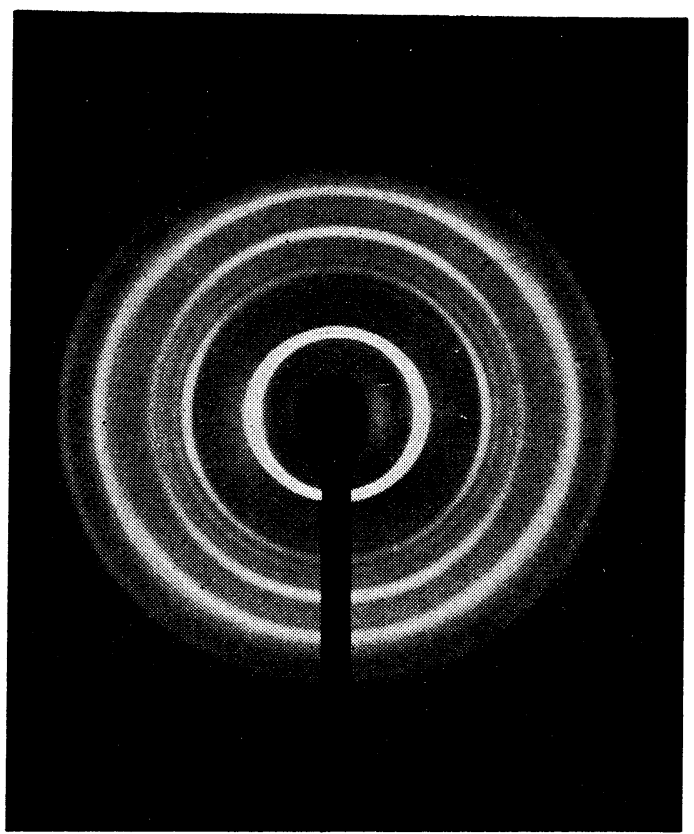

EV

Figure 3. Wide angle X-ray TV and EV photographs of PMDG solid film cast from a solution in chloroform (Group 1) and annealed at $488 \mathrm{~K}$ for $1 \mathrm{hr}$. 


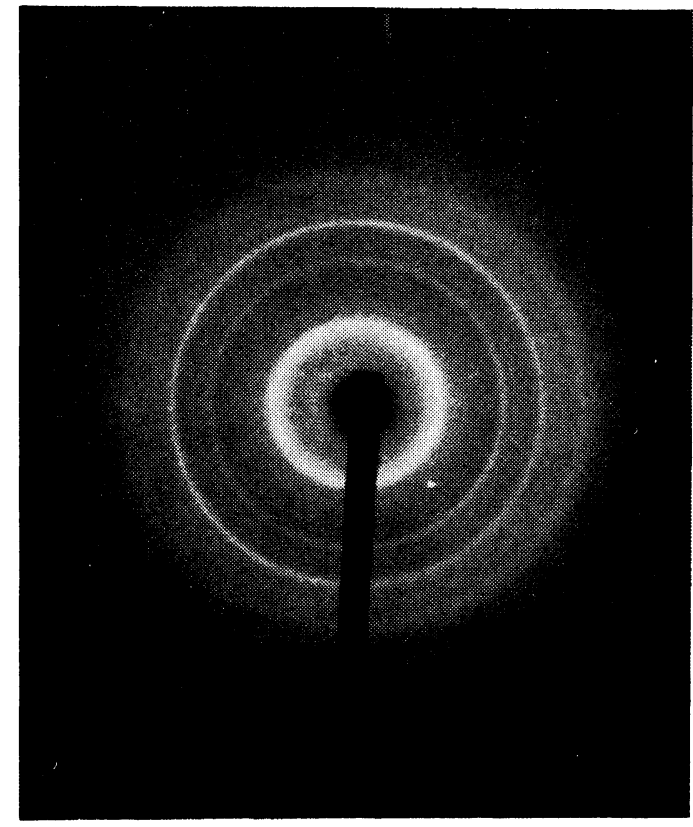

TV

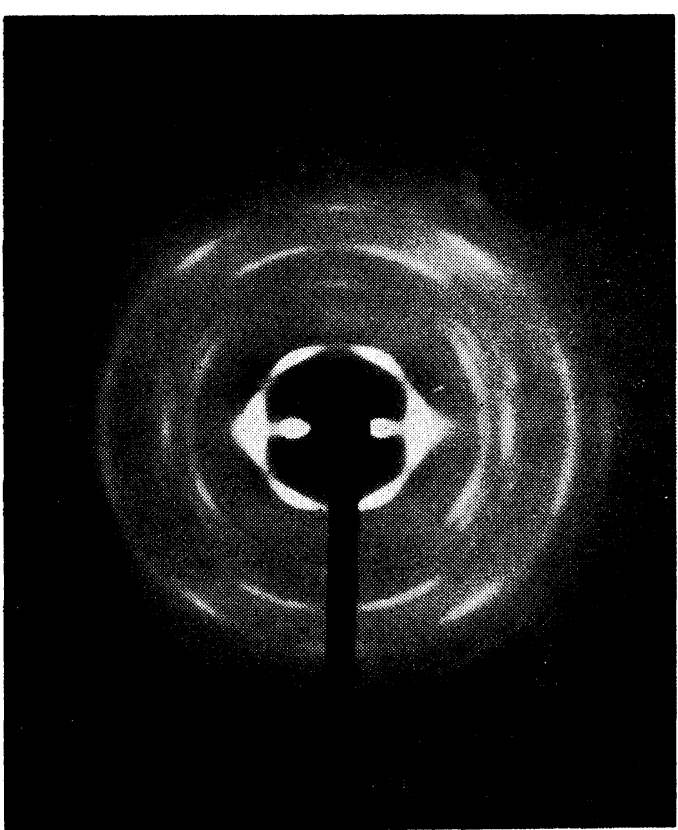

EV

Figure 4. Wide angle X-ray TV and EV photographs of PMDG solid film cast from a solution in trichloroethylene (Group 2) and annealed at $488 \mathrm{~K}$ for $1 \mathrm{hr}$.

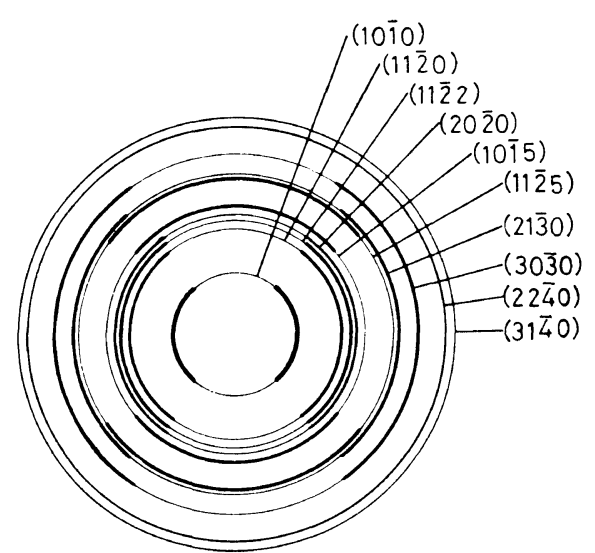

Observed X-ray EV Diagram

Figure 5. Indices of the observed X-ray EV diagram for PMDG film cast from a solution in chloroform (Group 1). The film surface is positioned along the vertical direction.

of crystallites composed of the $\alpha$-helical chains.

Figure 6(a) exhibits the situation of the molecular chain arrangement or the crystalline orientation represented by the three characteristic parameters, in which $\chi$ is the rotational angle of the $\alpha$-helical axis around the axis perpen- dicular to the film sulface, $\varphi$ is the inclination angle of the $\alpha$-helical axis from the surface plane, and $\omega$ is the rotational angle of the crystallite around the $\alpha$-helical axis. Figure 6 (b) shows these rotational parameters in the reciprocal space. They are designated by means of the Ewald's sphere (the large circle) and the diffraction sphere (the small circle), in which the surface of the film is positioned parallel to the $\mathrm{Y}-\mathrm{Z}$ plane. The $\mathrm{C}^{*}$ vector represents the $\alpha$-helical axis in the crystallite. The molecular chain orientation is determined by using these three rotational parameters so as to satisfy the X-ray diffraction patterns along the TV and EV directions. The Debye rings in the TV photograph of Figure 3 predict that the crystallite axis is freely rotational around the X-axis. Since not the sharp X-ray diffraction spots but the broad arcs are observed in the EV photograph, despite the hexagonal packing of the $\alpha$-helical chains in the PMDG crystallite, $\omega$ may rotate freely around the crystallite axis. From the angular measurement of extent of the arcs, the magnitude of inclination of the $C^{*}$ vector from the film surface, $\varphi$, is evaluated by comparing 


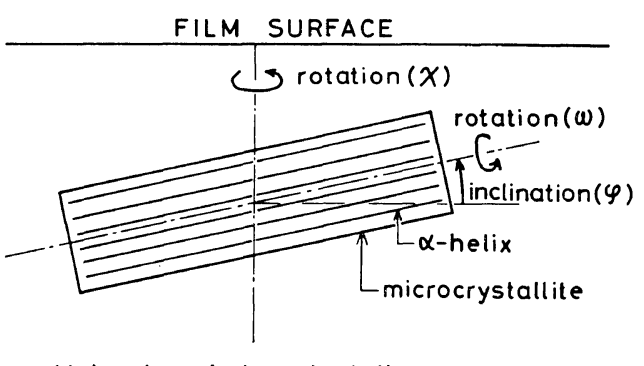

Molecular chain orientation

(a)

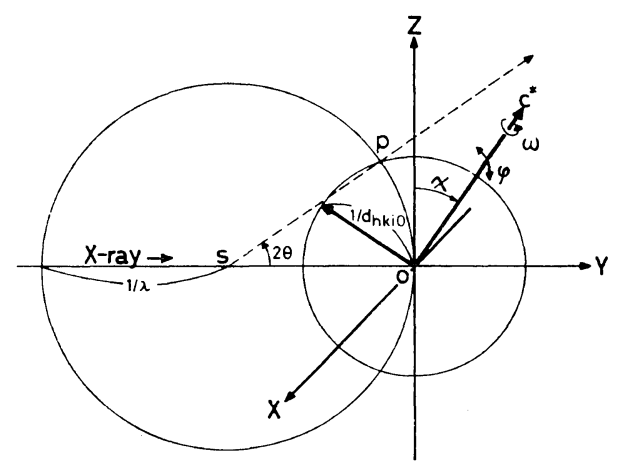

(b)

Figure 6. (a) Schematic representation of molecular chain orientation in crystallite and rotational parameters of $\chi, \omega$, and $\varphi$. (b) Reciprocal spacerotational parameter relationship when the film surface is parallel to the $\mathrm{Y}-\mathrm{Z}$ plane. The $\mathrm{X}$ axis is normal to $Y$ and $Z$ axis and perpendicular to the film surface. The large circle, small circle, and $C^{*}$ are Ewald's sphere, diffraction sphere and $\alpha$-helical axis in crystallite, respectively.

the diffraction patterns calculated for various angle of $\varphi$ with the experimental ones. Supposing that $\varphi$ is zero and, $\omega$ and $\chi$ rotate randomly around the $\mathrm{C}^{*}$-axis and the $\mathrm{X}$-axis, respectively, the density distribution of the reciprocal lattice points occurres on the diffraction sphere in Figure 6(b). That is, the density of the reciprocal lattice points of $\{\mathrm{hki0}\}$ is maximal on the $\mathrm{X}$-axis and it decreases on leaving the $\mathrm{X}$-axis. Therefore, the X-ray EV pattern of \{hki0\} shows arcs. In the case of $\{$ hkil\}, the $\mathrm{EV}$ profile of arcs is obtained in the same way as $\{$ hki0 $\}$. When $\varphi$ is not zero, each diffraction arc extends only along the Debye ring depending upon the distribution of $\varphi$. Evaluation of $\varphi$ is left for further study, such as the pole figure technique. Furthermore, the crystallites aggregate with a uniform distribution around the axis normal to the film surface and also, around the axis of the crystallites in which the $\alpha$-helical molecular chains are packed in the hexagonal array.

Next we will consider the aggregated states of the $\alpha$-helical molecular chains for the PMDG films cast from solutions belonging to Group 2 listed in Table I. The X-ray EV patterns for these samples are quite different from those of Group 1, as shown in Figures 3 and 4, though the TV patterns for Groups 1 and 2 are similar to each other. The sharp Debye rings in the TV photograph of Figure 4 confirm the existence of crystalline phase and their spacings correspond to the hexagonal array of the $\alpha$-helices. The EV photograph exhibits the six-spot diffraction pattern which satisfies for the most part the crystallographic constants of $a=b=1.19 \mathrm{~nm}$ and $c=2.7 \mathrm{~nm}$ with the hexagonal array ${ }^{9}$ as shown in Figure 7(a) and Table II. Figure 7(a) corresponds to the schematic diagram for the case in which the film surface is positioned along the vertical direction. The molecular chain arrangement of the PMDG films cast from solutions belonging to Group 2 can also be explained using the rotational parameters of $\chi, \omega$, and $\varphi$ in Figure 6. Supposing that the inclination angle of the $\alpha$-helical axis from the film surface, $\varphi$ is approximately zero, the combinations of $\chi$ and $\omega$ are classified into four cases: (1) both $\chi$ and $\omega$ are freely rotational, (2) $\chi$ is freely rotational and $\omega$ is restricted, (3) $\chi$ is restricted and $\omega$ is freely rotational, and (4) both of them are restricted. From the Debye rings of the TV photograph, the molecular chains around the axis perpendicular to the film surface are uniformly distributed and this corresponds to $\chi=$ $0^{\circ}-360^{\circ}$ (freely rotational). The arcs appear as the diffraction pattern of the EV photograph is both parameters are freely rotational. Therefore, the second case is the most desirable combination which can be used to successfully explain the patterns of the TV and EV photographs of Figures 4 and $7(a)$. That is, from the six-spot diffraction pattern of the EV photograph, the rotation of the crystallite around the $\alpha$ helical axis, $\omega$ and the inclination of the $\alpha$-helical axis from the film surface, $\varphi$ are both restricted. 


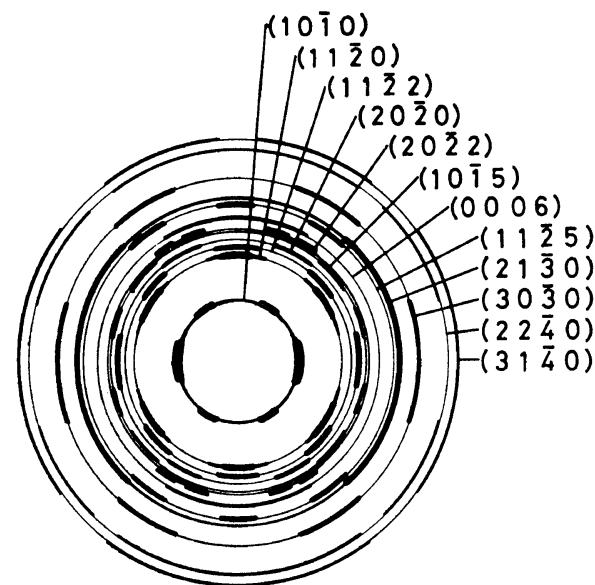

(a) Observed X-ray EV diagram of PMDG (Group 2)

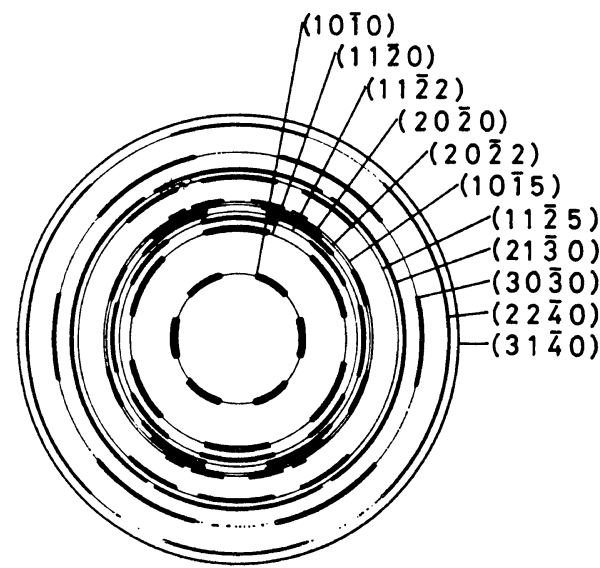

(b) Calculated X-ray EV diagram of PMDG (Group 2)

Figure 7. (a) Observed X-ray EV diagram for PMDG film cast from a solution in trichloroethylene (Group 2). The film surface is positioned along the vertical direction. (b) Calculated X-ray EV diagram corresponding to Figures 4 and 7 (a) (Group 2). Rotational parameters of $\chi=0^{\circ}-360^{\circ}$, $\omega=-18^{\circ}-18^{\circ}$, and $\varphi=0^{\circ}$ were used for calculation.

Table II. Spacings and intensities of X-ray

diffraction spots for PMDG film cast and dried from a solution in trichloroethylene and annealed at $488 \mathrm{~K}$ for $1 \mathrm{hr}$

\begin{tabular}{cccccc}
\hline $\begin{array}{c}d_{\text {obs }}, \\
\mathrm{nm}\end{array}$ & $\begin{array}{c}d_{\text {calcd }}, \\
\mathrm{nm}^{7}\end{array}$ & $I_{\text {through }}$ & $I_{\text {edge }}$ & $I_{\text {end }}$ & Index \\
\hline 1.034 & 1.036 & vs & vvs & vvs & $(10 \overline{1} 0)$ \\
0.598 & 0.598 & w & w & m & $(11 \overline{2} 0)$ \\
0.517 & 0.518 & - & vw & w & $(20 \overline{2} 0)$ \\
0.390 & 0.391 & w & m & s & $(21 \overline{3} 0)$ \\
0.345 & 0.345 & vw & w & w & $(30 \overline{3} 0)$ \\
0.296 & 0.299 & - & vw & vw & $(22 \overline{4} 0)$ \\
0.285 & 0.288 & - & vw & vw & $(31 \overline{4} 0)$ \\
0.553 & 0.547 & vw & vw & w & $(11 \overline{2} 2)$ \\
0.486 & 0.484 & - & vw & w & $(20 \overline{2} 2)$ \\
0.481 & 0.479 & $\mathrm{~m}$ & $\mathrm{~m}$ & - & $(10 \overline{1} 5)$ \\
0.402 & 0.401 & w & m & - & $(11 \overline{2} 5)$ \\
0.440 & $0.450^{\mathrm{a}}$ & $\mathrm{vw}$ & $\mathrm{vw}$ & - & $(0006)$ \\
\hline
\end{tabular}

a A. Elliott, poly $(\alpha$-amino acid $)$ s, G. D. Fasman, Ed., Marcel Dekker, New York, 1967, p 1.

On the assumption that the crystallites aggregate with a uniform distribution around the $\mathrm{X}$-axis with $\omega=-18^{\circ}-18^{\circ}$, the standard being the (1010) uniplanar orientation and $\varphi=0^{\circ}$, the schematic EV pattern is obtained as shown in Figure $7(\mathrm{~b})$, making consideration of the relationships in Figure 6(b). If $\omega$ is fixed to zero,

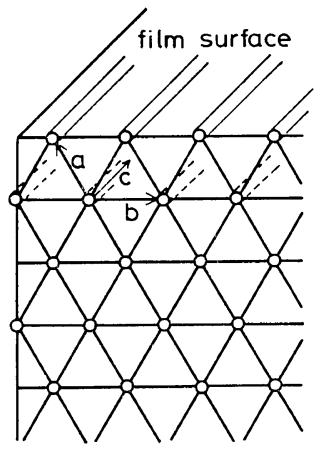

(a)

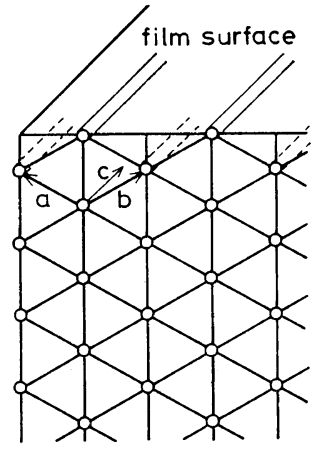

(b)

Figure 8. Two typical packing states of the $\alpha$ helical molecular chains: (a) the (1010) plane is

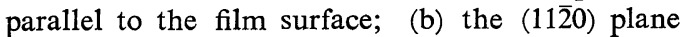
is parallel to the film surface (ref 3 ).

the expected EV diffraction pattern is inconsistent with the observed X-ray EV pattern even when $\varphi$ changes from $-10^{\circ}$ to $10^{\circ}$. In this case, the angles at the circumference of the arcs in the two figures are different from each other at a high Bragg angle. Therefore, it is apparent that $\omega$ is not fixed to zero but has an angular distribution. For the case of $\omega=-18^{\circ}-18^{\circ}$ and $\varphi=-10^{\circ}-10^{\circ}$, the angles at the circumference of arcs in a calculated EV pattern are slightly larger than those in the experimentally 
obtained pattern with respect to the diffractions from (hki0). Consequently, the combination of $\varphi=0^{\circ}$ and $\omega=-18^{\circ}-18^{\circ}$ is said to be fairly favorable. Robinson, et al., ${ }^{3}$ reported two characteristic cases for the partially hexagonal arrangement of the $\alpha$-helical rods in a concentrated solution of polypeptide, in which the (10ī0) and the $(11 \overline{2} 0)$ planes are parallel to the film surface as shown in Figures $8(\mathbf{a})$ and $8(\mathbf{b})$, respectively. These molecular chain alignments mean that $\omega$ is fixed and $\varphi$ is almost $0^{\circ}$. In an extreme case in which $\omega$ is zero, the $(10 \overline{1} 0)$ plane is parallel to the film surface, corresponding to the so-called uniplanar orientation as shown in Figure 8(a). The calculated EV pattern of Figure $7(b)$ is in qualitatively good agreement with Figures 4 and 7(a). This result indicates that the $\alpha$-helical molecular chains in PMDG cast from solutions belonging to Group 2 aggregate to form the uniplanar orientation of (1010) along the film surface.

The two characteristic patterns of the X-ray EV photographs as shown in Figures 3 and 4 may be explained respectively by the existence

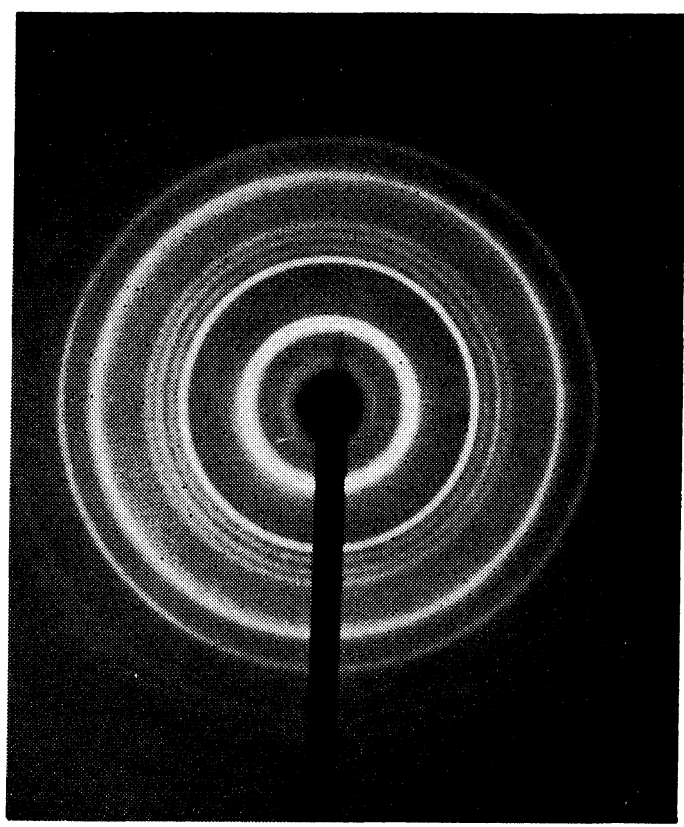

(a) and the absence of the $\omega$-rotation of the crystallite around the $\alpha$-helical axis. Further, these planar and uniplanar orientations can be confirmed by the X-ray end view photograph (End V) for the stretched samples of the PMDG films cast from solutions in chloroform (Group 1) and trichloroethylene (Group 2). Figure 9 shows the End V photographs for these specimens, of which the film surface is positioned along the vertical direction. Since the $\alpha$-helical axes align principally along the stretching direction, the End $\mathrm{V}$ diffraction pattern directly shows, the projection of the $a-b$ plane, that is, the distribution of $\omega$, if the packing state of crystallites does not change by stretching except for the rotation about the axis perpendicular to the film surface. Figure 9 shows Debye rings for the film cast from a solution in chloroform (Group 1) and the six-spot diffraction pattern for that in trichloroethylene (Group 2). These results confirm that crystallites aggregate with a uniform distribution about the $\alpha$-helical axis for Group $1\left(\omega=0^{\circ}-360^{\circ}\right)$ and with approximately fixed orientation for Group $2(\omega= \pm$ several

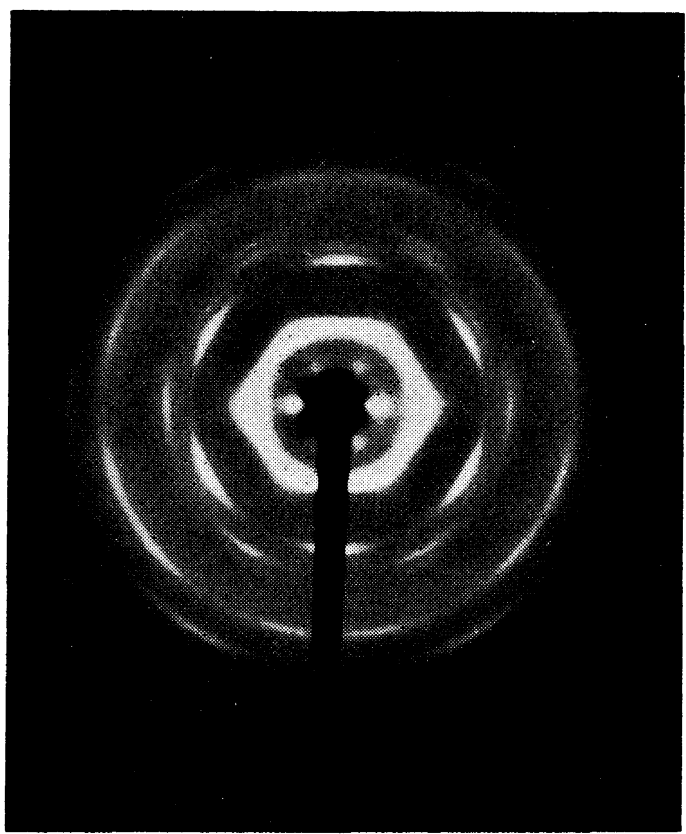

(b)

Figure 9. Wide angle X-ray End V photographs of drawn PMDG films cast from solutions in chloroform (a), and trichloroethylene (b), respectively. Drawing temperature is $453 \mathrm{~K}$. Drawing ratios are (a) $200 \%$ and (b) $163 \%$. The film surface is positioned along the vertical direction. 
degrees), forming the uniplanar orientation of the (1010) plane.

Solution Viscosity of Poly( $\gamma$-methyl D-glutamate)

Figure 10 shows a plot of the viscosity of various solutions of PMDG against their polymer concentration at $313 \mathrm{~K}$, in which the solvents indexed from 1 to 3 and from 4 to 6 belong to Groups 1 and 2, respectively. The solution viscosity of Group 1 exhibits the discontinuity of the slope at about 10 wt $\%$ which is regarded as the A-point. The A-point can be approximately estimated by using Flory's equation, ${ }^{4}$

$$
v^{*}=(8 / x)(1-2 / x)
$$

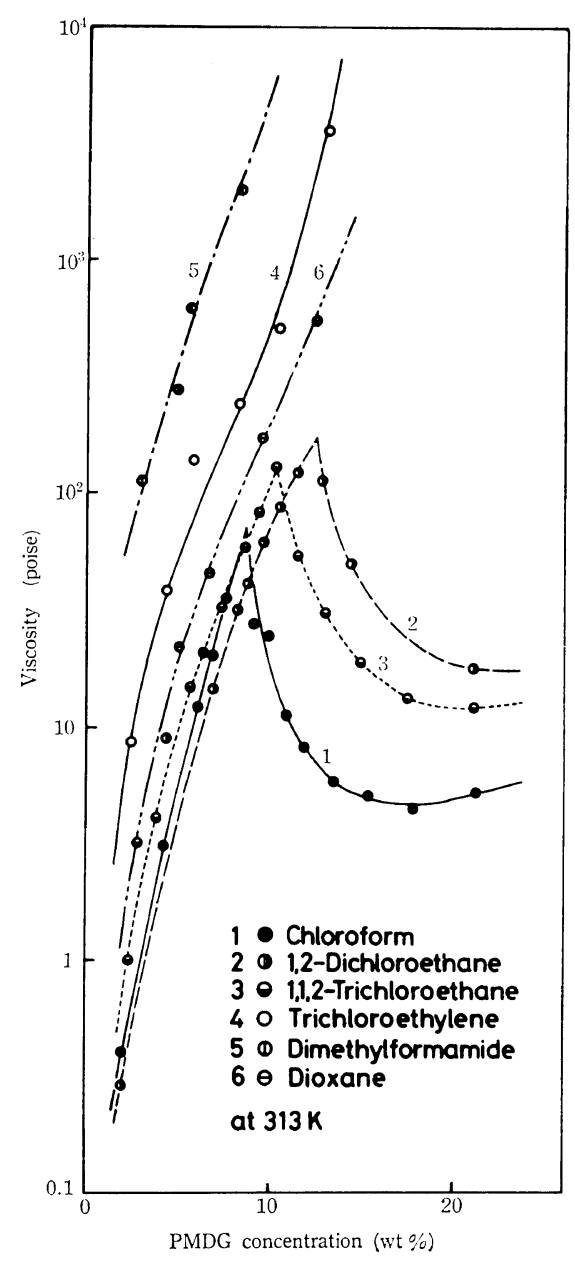

Figure 10. A plot of viscosity against polymer concentration in various PMDG solutions. The measurements were carried out at $313 \mathrm{~K}$. where $v^{*}$ is the volume fraction of PMDG at the A-point and $x$ is the axial ratio of PMDG. $x$ is calculated to be 77 from the length of helical PMDG used $(147 \mathrm{~nm})$ and its diameter of $1.9 \mathrm{~nm}$ on the assumption that the side chains are extended. $v^{*}$ turned out to be 0.10 . The critical weight fraction observed is very close to this value, because the densities of solvent $(\rho=1.26-1.48)$ and PMDG $(\rho=1.28-1.29)$ are very close to each other. The calculated concentration for phase separation (A-point) shows good agreement with the experimental one shown in Figure 10. Decrease of viscosity with increasing concentration above the concentration of $10 \mathrm{wt} \%$ is due to the formation of an anisotropic phase. As expected from the concentration dependence of viscosity, the liquid crystalline phase is formed at $313 \mathrm{~K}$ in the solutions belonging to Group 1 tabulated in Table I. Figure 10 also shows that the discontinuity or decrease in the concentration dependence of viscosity is not observed in the solutions belonging to Group 2 (curves 4-6). This fact may indicate that the liquid crystalline phase is not formed at $313 \mathrm{~K}$ in these solutions belonging to Group 2. The aggregated states of the $\alpha$-helical molecular chains in the solid film seem to depend on the formation of the liquid crystalline phase in a concentrated solution. The planar orientation is observed for the PMDG film cast passing through a liquid crystalline state in a concentrated solution belonging to Group 1 . Also, the (1010) uniplanar orientation is observed for the specimen cast from a concentrated solution of Group 2 without the observation of the formation of the liquid crystalline phase. As mentioned above, the aggregated states of molecular chains in the solid film are strongly affected by those in solution. The appearance of the liquid crystalline state can be investigated by the observations of concentrated solutions under a polarizing optical microscope, as will be mentioned in the next section.

\section{Optical Microscopic Observation of Liquid Crys- talline State}

Various solutions are poured into the glass cell and observed under the cross nicols at room temperature. Figure 11(a) shows the polarizing micro-photograph of a PMDG solution of 21- 


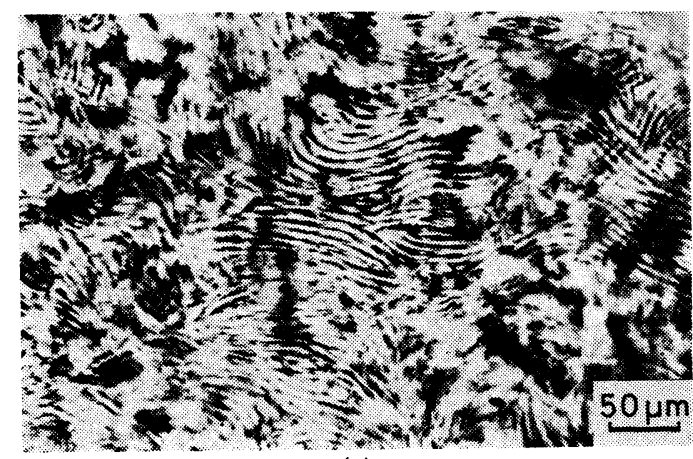

(a)

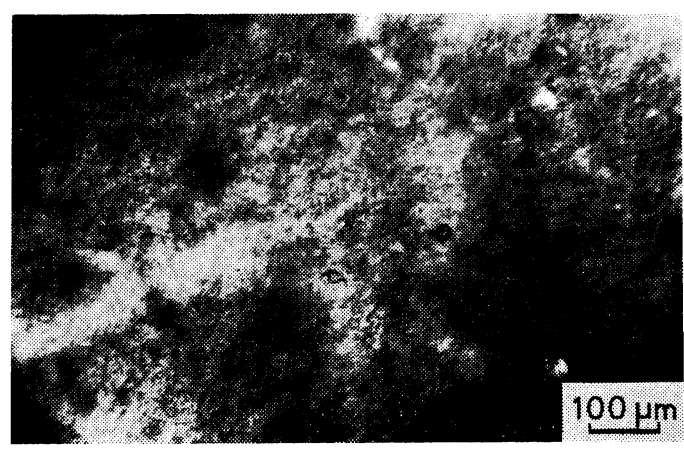

(b)

Figure 11. (a) Polarizing optical micro-photograph of a 21-wt \% PMDG solution in chloroform (Group 1). Retardation lines and iridescent color were observed at room temperature. (b) Polarizing optical micro-photograph of a 18-wt $\%$ PMDG solution in $N, N$-dimethylformamide (Group 2). Observation was carried out at room temperature.

wt $\%$ in chloroform (Group 1). The observation under the optical microscope was carried out by using the glass cell having a Teflon spacer with a thickness of $1.1 \mathrm{~mm}$. In this case, the cholesteric axis is formed parallel to the surface of the glass plate. The picture of Figure 11 was taken by transmitting the light perpendicular to the glass plate. Iridescent color and retardation lines corresponding to the half interval of one pitch characteristic of the cholesteric liquid crystal are observed. These results are consistent with the results for poly $(\gamma$-benzyl L-glutamate) observed by Robinson, et al. ${ }^{3}$ The presence of iridescent color and retardation lines is in close relation to the concentration dependence of viscosity, and is the evidence for the anisotropic phase of the cholesteric twisted structure above the A-point. On the other hand, the solution belonging to Group 2 looks like a gel at high concentrations above the A-point. Figure 11(b) exhibits the polarizing micro-photograph of a PMDG solution of 18 -wt $\%$ in $N, N$-dimethylformamide (Group 2). Though bright portions are observed under a polarizing microscope, there are no retardation lines and the iridescent color characteristic of the cholesteric liquid crystalline phase in a field of vision. The existence of these bright portions may be explained as the formation of anisotropy in the gel phase and/or by the residue of a cholesteric phase in the gel during cooling the solution of Group 2 to room temperature. It may not be unreasonable to consider that concentrated solutions of Group 2 are composed of the gel phase at room temperature because the retardation lines were not observed under the polarizing optical microscope. On the basis of the X-ray diffraction studies, the optical microscopic observations and the viscosity measurements, the aggregated states of molecular chains in the solid-state films seem to be strongly affected by those of the solution-states. That is, solutions belonging to Group 1 pass through the state of cholesteric liquid crystal in the process of solidifying, whereas those belonging to Group 2 do not show such process around room temperature. When the solid film is prepared by evaporation of solvents belonging to Group 2 at a higher temperature at which a gel-like state changes into a solution state, the wide angle X-ray EV photograph of this specimen exhibits a similar pattern of arcs to that of Group 1. The solid film indicates the planar orientation. The gel formation may be one of the important processes for the formation of uniplanar orientation in the solid-state, though the mechanism from a gel-state to the molecular aggregation in the solid still needs further study.

Next we should like to try to speculate on the factors which determine the type of molecular chain aggregation in the solid-state. Formation of the planar and the uniplanar orientations may be attributed to the shape of the crystallite. If the crystallites take the form of a thin platelet in the evaporation process of solvents belonging to Group 2, the stacking of the thin platelets easily induces the uniplanar orientation, though 
it is not apparent whether the molecular chain dispersion in solvents of Group 2 is in optimum condition to form the lamellar shape crystallite or not. Although the mechanism favorable to the formation of the $(10 \overline{1} 0)$ uniplanar orientation parallel to the film surface of PMDG (Group 2) is not clear, the variation of the uniplanar orientation can be used to explain the different crystal growth rates along [1010] and [1120]. These two uniplanar orientations are very likely in the case of hexagonal packing. If the crystal

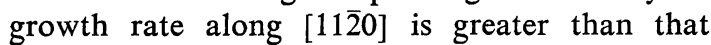
along [1010], the thin platelet of crystallite stacks along [1010] nicely. This is the condition for the PMDG cast from solutions in Group 2 solvents, indicating the selective (1010) uniplanar orientation. While the mechanism generating the actual aggregated state of PMDG crystallites has not been directly proved, the reason mentioned above is a reasonably good speculation.

The experimental results obtained by using the X-ray diffraction studies, the polarizing optical microscopic observations, the viscosity measurements and the observations of gel formation with respect to the aggregated state of the $\alpha$-helical molecular chains of PMDG are summarized in the following. The planar orientation in a solid film is formed by passing through the cholesteric-like liquid crystalline state in a concentrated solution belonging to Group 1 . The (1010) uniplanar oriention is formed by passing through a gel state in a concentrated solution belonging to Group 2. The stripes of the retardation lines observed by a polarizing optical microscope are found to be almost parallel to the substrate of the cell or the drying film surface. Hereupon it should be noticed that the relationship between the directions of the optical microscopic observation of Figure 11 is consistent with that of the X-ray edge view photograph of Figure 3. Therefore, in a concentrated solution the cholesteric axis is approximately perpendicular to the solution surface. The size of the crystallites along [hkil] is evaluated following Hosemann's paracrystalline analysis. ${ }^{10}$ The morphological observation reveals that the aggregated structure of PMDG is decomposed into small crystalline blocks. ${ }^{11}$ The lateral dimensions of the crystallites evaluated on the basis of Hosemann's paracrystalline analysis and the morphological observation are comparable to each other. These results indicate that the solid-state of PMDG is composed of the crystalline blocks with the lateral size of $20-50 \mathrm{~nm}$. Figure 12 shows the expected solidifying process accompanying the variation of aggregated state of the $\alpha$-helices when the solid film is produced from a con-

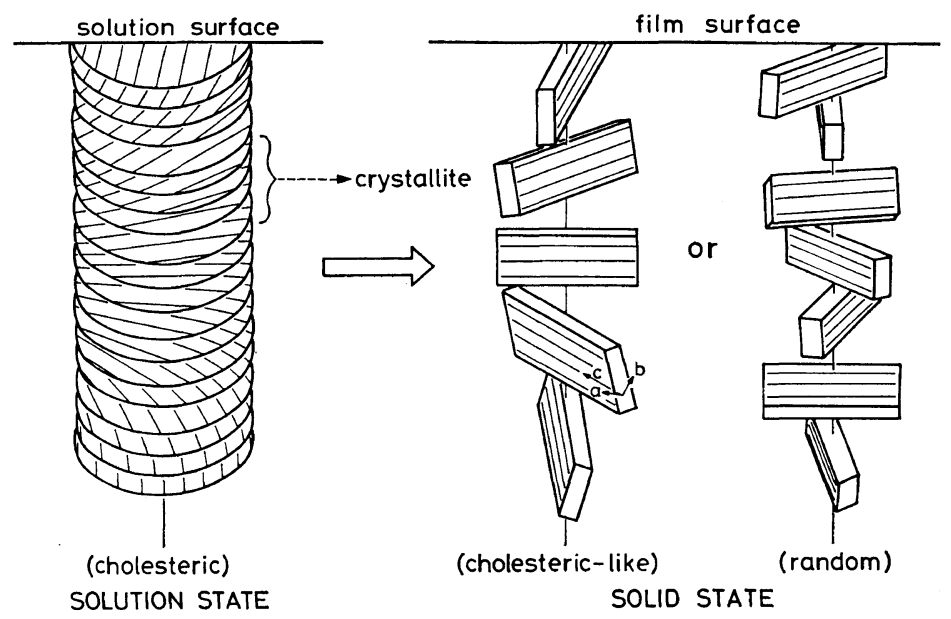

Figure 12. Schematic representation of aggregated states $\alpha$-helical molecules in a concentrated solution (cholesteric liquid crystal) and solid-state film (cholesteric-like and/or random aggregation of crystallites). Parallel straight lines represent $\alpha$-helical molecular chains. 
centrated solution. It seems reasonable to consider that several cholesteric planes within a certain region in the cholesteric structure are piled up, reducing the small helical twist and aligning their $\alpha$-helical axes to form the crystallites. Two ways for the aggregated states of crystallites are possible: cholesteric-like structure and random structure as shown in Figure 12. The definite aggregated structure of crystallites can not be determined by X-ray $\mathrm{TV}$ and $\mathrm{EV}$ patterns. At the present, the classification of solvents such as those of Groups 1 and 2 is based only on the types of the molecular chain aggregation observed experimentally in a solution or a solid-state at room temperature. The effect of the size and the dielectric constant of the solvent molecule, the polymer-solvent interaction and so on with respect to the molecular chain aggregation require further investigation.

\section{Aggregated State and Orientation of Molecular}

Chains in Poly( $\gamma-n$-hexyl D-glutamate)

The solid films of poly $(\gamma-n$-hexyl D-glutamate $)$ (PnHDG) with long side chains, $-\mathrm{CH}_{2} \mathrm{CH}_{2} \mathrm{COO}$. $\left(\mathrm{CH}_{2}\right)_{5} \mathrm{CH}_{3}$, are prepared from solutions in

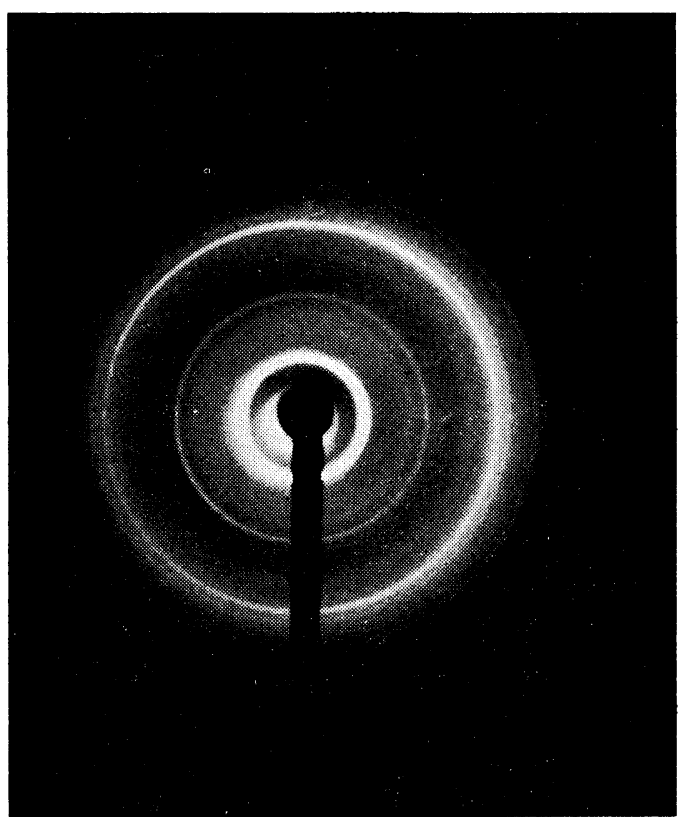

TV chloroform, 1,2-dichloroethane, trichloroethylene, or benzene as shown in Table III. Figure 13 shows the X-ray photographs of the through view (TV) and the edge on view (EV) for the film cast from a chloroform solution. The sample is fixed so that its surface plane is in the vertical position for the photograph of the EV. The fundamentally similar TV and EV patterns for the films from the other solvents listed in Table III are observed. In contrast to the case of PMDG, a feature of the EV patterns of the solid films is that they do not depend on the kind of solvents used (Groups 1 and 2). All diffraction spots seen in Figure 13 satisfy the crystallographic constants of $a=b=$ $1.59 \mathrm{~nm}$ and $c=2.7 \mathrm{~nm}$ of hexagonal packing,

Table III. Solvents used for poly $(\gamma-n$-hexyl D-glutamate)

\begin{tabular}{lcl}
\hline \multicolumn{1}{c}{ Solvents } & $\begin{array}{c}\text { Dielectric } \\
\text { constants }\end{array}$ & Orientation \\
\hline Chloroform & 4.8 & \\
1,2-Dichloroethane & 10.4 & Uniplanar \\
Trichloroethylene & 3.4 & Orientation \\
Benzene & 2.3 & \\
\hline
\end{tabular}

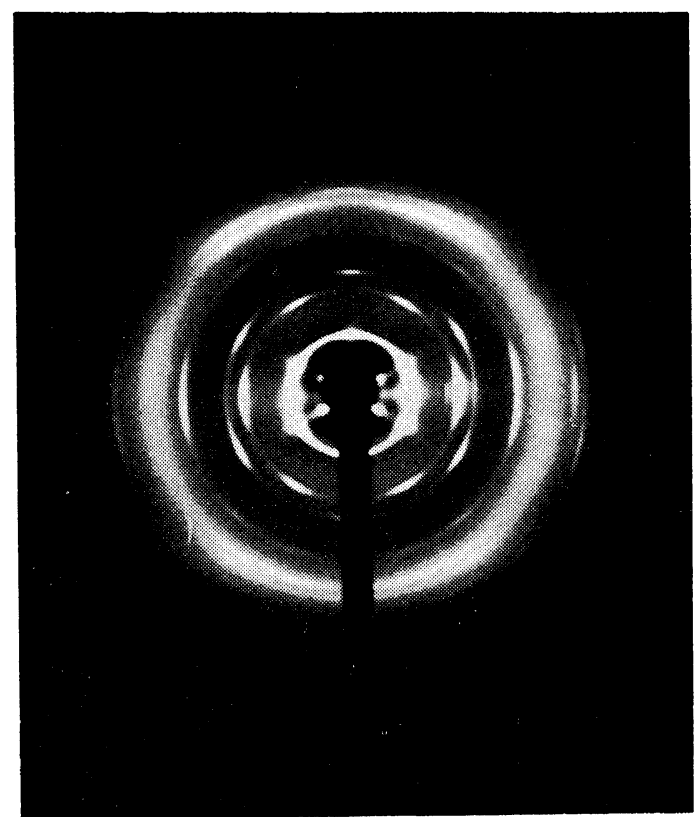

EV

Figure 13. Wide angle X-ray TV and EV photographs of PnHDG cast from a solution in chloroform. The film surface is positioned along the vertical direction. 
Table IV. Spacings and intensities of X-ray diffraction spots for PnHDG film cast and dried from a solution in chloroform

\begin{tabular}{ccclll}
\hline $\begin{array}{c}d_{\text {obs }}, \\
\mathrm{nm}\end{array}$ & $\begin{array}{c}d_{\text {calcd }}, \\
\mathrm{nm}\end{array}$ & $I_{\text {through }}$ & $I_{\text {edge }}$ & $I_{\text {end }}$ & Index \\
\hline 1.375 & 1.376 & vvs & vvs & vvs & $(10 \overline{1} 0)$ \\
0.795 & 0.794 & vw & m & m & $(11 \overline{2} 0)$ \\
0.688 & 0.688 & w & m & m & $(20 \overline{2} 0)$ \\
0.521 & 0.519 & vw & m & m & $(21 \overline{3} 0)$ \\
0.397 & 0.398 & - & vw & vw & $(22 \overline{4} 0)$ \\
0.381 & 0.381 & w & w & w & $(31 \overline{4} 0)$ \\
0.344 & 0.344 & - & vw & vw & $(40 \overline{4} 0)$ \\
0.316 & 0.315 & - & vw & vw & $(32 \overline{5} 0)$ \\
0.301 & 0.300 & - & vw & vw & $(41 \overline{5} 0)$ \\
0.499 & 0.502 & - & vw & - & $(10 \overline{1} 5)$ \\
0.449 & 0.447 & m & m & vw & $(11 \overline{2} 5)$ \\
0.427 & 0.425 & vw & w & vw & $(20 \overline{2} 5)$ \\
\hline
\end{tabular}

whose indices and spacings are shown in Table IV. Sharp Debye rings and spots in the TV and the EV photographs indicate the existence of the crystalline phase. The six-spot diffraction pattern of the EV photograph indicates that the $\alpha$-helical molecular chains are packed in a uniplanar orientation form, similar to that for the PMDG film cast from a solution belonging to Group 2. The aggregated state of the crystallites of PnHDG can also be explained in a similar manner as mentioned for the PMDG, using the rotational parameters of $\chi, \omega$, and $\varphi$ defined in Figure 6. From the shape of the Debye rings of the TV pattern, the crystallites composed of the $\alpha$-helical chains are known to aggregate with a uniform distribution about the axis perpendicular to the film surface (the rotation is with respect to $\chi$ ). Rotation of the crystallites around the $\alpha$-helical axis and inclination of the crystallites from the film surface are considerably restricted. Here, we should notice that the EV diffraction spots of PnHDG rotate by $30^{\circ}$ in comparison with those of the corresponding reflection planes of PMDG. This indicates that the uniplanar orientation of the $(11 \overline{2} 0)$ plane is formed in the PnHDG film cast from solutions belonging to both Groups 1 and 2. When the molecular chain axes lie in the film surface $\left(\varphi=0^{\circ}\right)$ and the (1120) plane slightly rotates about the molecular chain axis $\left(\omega=-10^{\circ}-10^{\circ}\right)$, the calculated diffraction pro- file is obtained as shown in Figure 14. This calculated diffraction pattern essentially resembles the experimentally obtained X-ray EV pattern of Figure 13. Existence of the (1120) uniplanar orientation is also comfirmed by the X-ray End V photograph, using the stretched specimen of PnHDG. Figure 15 shows the End $V$ pattern for which the PnHDG film surface is positioned in the vertical direction. Since the End $\mathrm{V}$ pattern corresponds to the projection of the $a-b$ plane, this pattern apparently confirms that the $(11 \overline{2} 0)$ planes are parallel to the film surface, and this is the so-called uniplanar orientation of the $(11 \overline{2} 0)$ plane.

As mentioned above, the aggregated states of $\alpha$-helices in PnHDG solid films cast from various solutions are not sensitive to solvents, though those for PMDG are remarkably sensitive to solvents for Groups 1 and 2. Figure 16(a) and (b) show the polarizing micro-photographs for a 20-wt $\%$ PnHDG solution in 1,2-dichloroethane (Group 1) and that of a 18-wt $\%$ solution in trichloroethylene (Group 2), respectively. In both cases the dark and bright stripes of the retardation lines and iridescent color characteristic of cholesteric liquid crystalline phase are observed. Since the $\alpha$-helical PnHDG molecules pass through the cholesteric liquid crystalline state in the process of solidifying, the cholesteric liquid crystal in a concentrated solu-

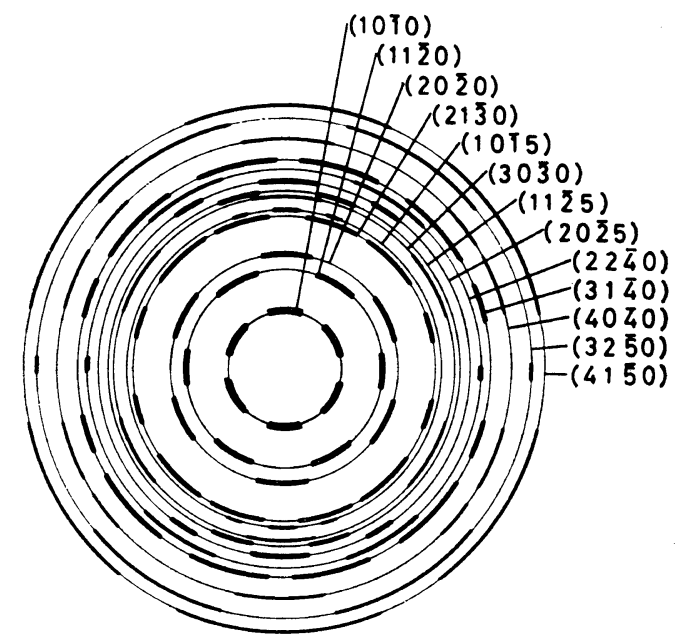

Figure 14. Calculated X-ray EV diagram of PnHDG. Rotational parameters of $\chi=0^{\circ}-360^{\circ}$, $\omega=-10^{\circ}-10^{\circ}$, and $\varphi=0^{\circ}$ were used for calculation. 


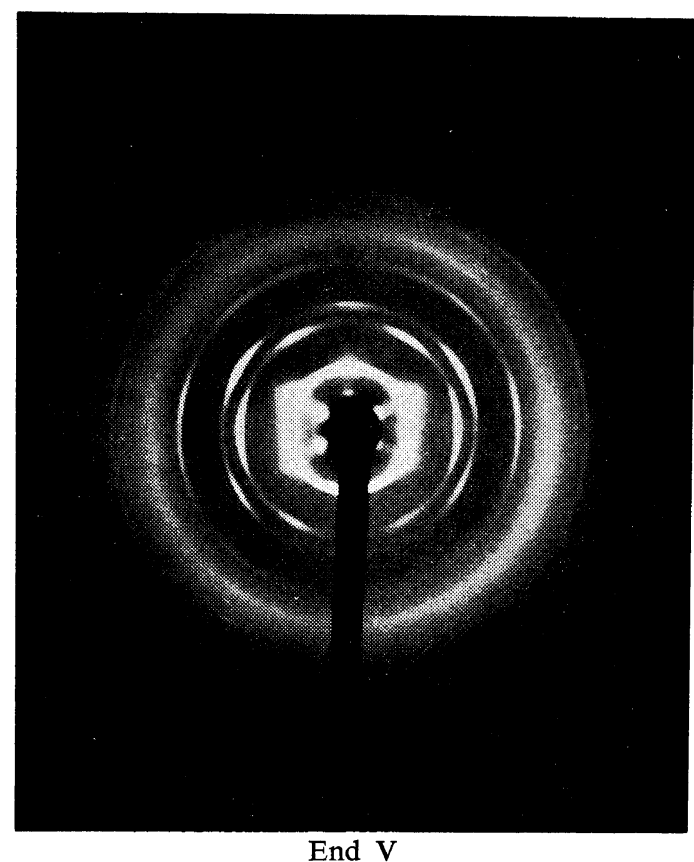

Figure 15. Wide angle $\mathrm{X}$-ray End V photograph of drawn PnHDG film cast from a solution in chloroform. Drawing temperature is $298 \mathrm{~K}$ and drawing ratio is $150 \%$. The film surface is positioned along the vertical direction.

tion may be reflected on the aggregated state of crystallites composed of $\alpha$-helices of PnHDG in a solid film prepared from it. The cholesteric planes composed of the $\alpha$-helices have a small twist relative to one another around the axis perpendicular to the planes and may be the cause of the aggregated states of crystallites along the direction perpendicular to the film surface in the manner of the (1120) uniplanar orientation as shown in Figure 12.

Though the cholesteric liquid crystal is formed in concentrated solutions of PMDG with Group 1 solvents and of PnHDG with Groups 1 and 2 solvents, their aggregated states or molecular orientations in the solid films are quite different. That is, the former is the planar orientation $\left(\chi=0^{\circ}-360^{\circ}\right.$ and $\left.\omega=0^{\circ}-360^{\circ}\right)$ and the latter is the $(11 \overline{2} 0)$ uniplanar orientation $\left(\chi=0^{\circ}-360^{\circ}\right.$, $\omega=-10^{\circ}-10^{\circ}$, and $\left.\varphi=0^{\circ}\right)$. This difference may arise from the shape of crystallites as described for the cases of PMDG films cast from Group 1 solvents and from Group 2 solvents

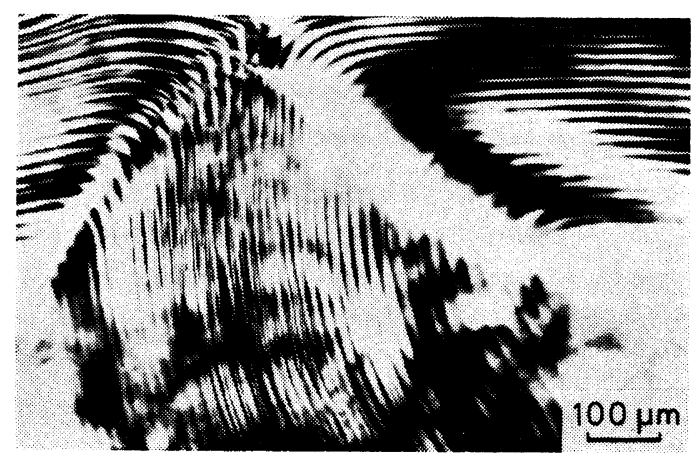

(a)

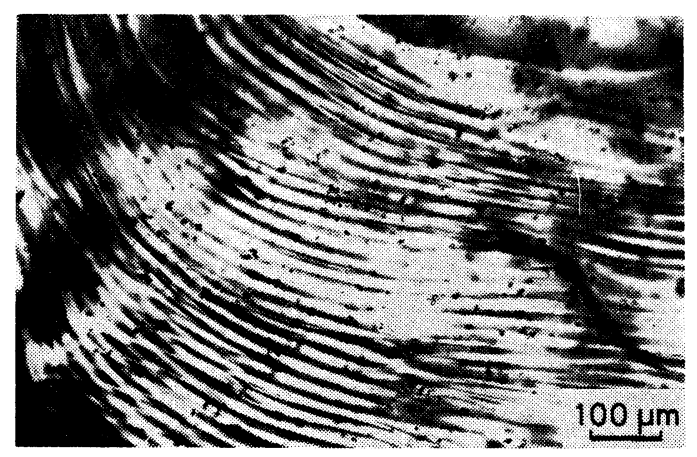

(b)

Figure 16. (a) Polarizing optical micro-photograph of a $20-w t \%$ PnHDG solution in 1,2-dichloroethane (Group 1), and (b) that of a 18-wt $\%$ solution in trichloroethylene (Group 2). Observations were carried out at room temperature.

in the previous section. If the growth rate of the crystallite along [10 $\overline{10}$ ] is assumed to be much greater than that along [1120] in the PnHDG crystallite and consequently, the crystallites in a shape of a thin platelet may favorably stack along [1120]. This thin plate may easily form the $(11 \overline{2} 0)$ uniplanar orientation. On the other hand, if the growth rates of the crystallites along [10̄0] and [1120] are comparable in PMDG solid films cast from Group 1 solvents, then the crystallites having the shape of a regular rectangular prism is preferably formed. These crystallites of a regular rectangular prism may aggregate aligning their longer axes in a certain plane by statistically random rotation around the $\alpha$-helical molecular chain axes. These speculations are to be confirmed by measuring the geometrical dimensions of the crystalline blocks. 


\section{CONCLUSIONS}

The aggregated states of molecular chains of PMDG in the solid film are remarkably sensitive to the kind of solvent from which the film is made. The aggregated states or the orientations of the $\alpha$-helical chains in a solid-state film are investigated by X-ray diffraction studies, the optical microscopic observations, the viscosity measurements and including the observation on the gel. The planar orientation is produced by passing through the formation of a cholesteric liquid crystal in a concentrated solution of PMDG belonging to Group 1 . The (1010) uniplanar orientation is produced by passing through the gel formation in a concentrated solution of PMDG belonging to Group 2. Since the cholesteric liquid crystalline phase is not observed in the solutions belonging to Group 2, the cholesteric-like aggregation of the crystallites can not necessarily be expected, but it is possible also that the crystallites randomly aggregate with a uniform probability about the axis perpendicular to the film surface. On the other hand, the $(11 \overline{2} 0)$ uniplanar orientation is produced through the formation of cholesteric liquid crystal in a concentrated solutions of PnHDG belonging to Groups 1 and 2. The orientation of the crystallites of PnHDG is not so sensitive to the kind of solvents used, unlike PMDG. Classification of solvents with respect to the aggregated state or the molecular chain orientation based on the magnitude of dielectric constants, the size of solvent, the polymer-solvent interaction and so on remains for further study.
It is realized that the mechanism for the molecular chain orientation proposed here is still in a stage of speculation as to the true aggregated state, but it is hoped that this will serve as a basis for further refinements concerning the elucidation of the relationship among the molecular chain aggregations produced in dilute solutions, concentrated solutions and in the solid film.

Acknowledgment. The authors offer their grateful acknowledgment to Ajinomoto Co., Ltd. for supplying them with poly( $\gamma$-methyl D-glutamate) samples.

\section{REFERENCES}

1. P. Doty, J. H. Bradbury, and A. M. Holtzer, J. Am. Chem. Soc., 78, 947 (1956).

2. A. Wada, J. Polym. Sci., 45, 145 (1960).

3. C. Robinson, J.C. Ward, and R. B. Beevers, Discuss. Faraday Soc., 25, 29 (1958).

4. P. J. Flory, Proc. Roy. Soc., A243, 73 (1956).

5. J. Hermans, Jr., J. Colloid Sci., 17, 638 (1962).

6. A. J. McKinnon and A. V. Tobolsky, J. Phys. Chem., 70 (1966).

7. E.M. Bradbury, B. G. Carpenter, and H. Goldman, Biopolymers, 6, 837 (1968).

8. M. Takayanagi and S. Kuriyama, Kogyo Kagaku Zasshi (J. Chem. Soc. Japan, Ind.), 57, 876 (1954).

9. C. H. Bamford, L. Brown, A. Elliott, W. E. Hanby, and I. F. Trotter, Nature, 169, 357 (1952).

10. R. Hosemann, Polymer, 3, 349 (1962).

11. H. Tanizaki, T. Kajiyama, and M. Takayanagi, Rep. Prog. Polym. Phys. Jpn., 18, 587 (1975). 